\title{
Numerically Enhanced Stimulated Emission Depletion Microscopy with Adaptive Optics for Deep-Tissue Super-Resolved Imaging
}

\section{Supporting Information}

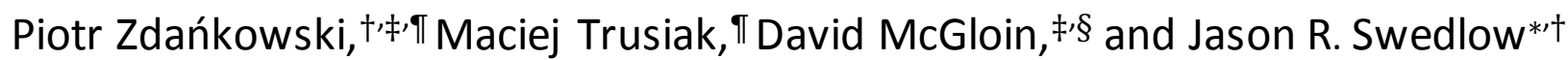

†Centre for Gene Regulation and Expression, School of Life Sciences, University of Dundee, Dundee, DD1 5EH UK

$\ddagger S U P A$, School of Science and Engineering, University of Dundee, Dundee, DD1 4HN UK

TWarsaw University of Technology, Institute of Micromechanics and Photonics, 8 A. Boboli St., 02-525 Warsaw, Poland

§School of Electrical and Data Engineering, University of Technology Sydney, Ultimo, NSW 2007, Australia

E-mail: jrswedlow@dundee.ac.uk

\section{Dual-SLM 3D STED microscope setup}

Our STED microscope schematic is presented in Fig. 1. 


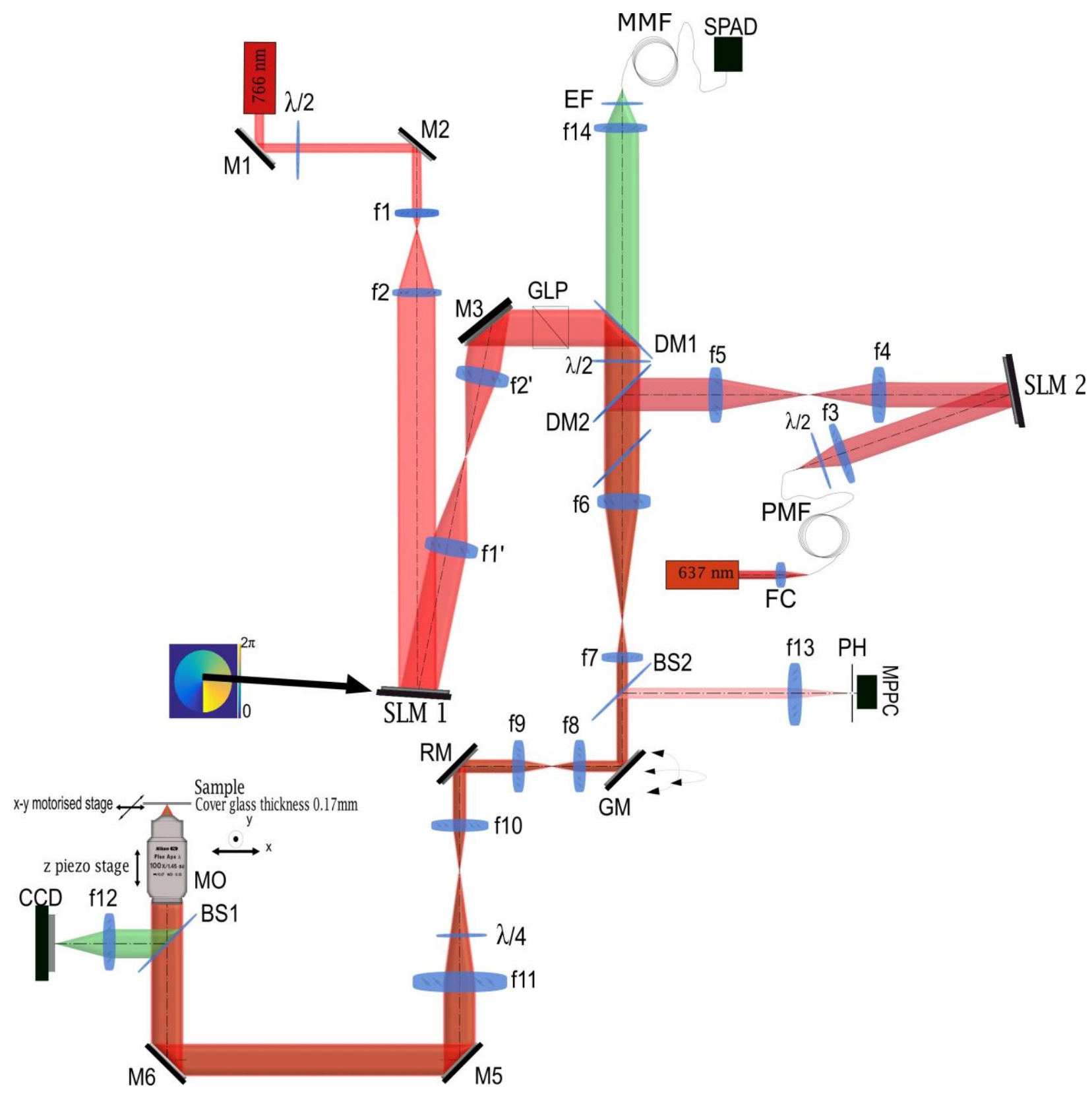

Figure 1: The optical setup of the custom built dual-SLM 3D STED microscope. 766nm - 766nm depletion laser, $637 \mathrm{~nm}-637 \mathrm{~nm}$ excitation laser, BS1-BS2 - pellicle beamsplitters, CCD - CCD camera, DM1-DM2 dichroic mirrors, EF - emission bandpass filter, f1-f14 - lenses, FC - fibre coupling lens, GLP - glan laser polarizer, GM - pair of galvanometric mirrors, $\lambda / 2$ - half-wave plate, $\lambda / 4$ - quarter-wave plate, M1-M6 - steering mirrors, MMF - multi-mode fibre, MO microscope objective, MPPD - multi pixel photon counter, PH - pinhole, PMF - polarization maintaining fibre, RM - resonant mirror, SLM 1 and SLM 2 spatial light modulators, SPAD - single photon avalanche diode. 


\section{Depletion beam path}

Depletion beam originates at the $766 \mathrm{~nm}$ laser (PicoQuant VisIR 765 STED). It can be set manually to work at $80 \mathrm{MHz}, 40 \mathrm{MHz}, 20 \mathrm{MHz}, 10 \mathrm{MHz}, 5 \mathrm{MHz}$, or $2.5 \mathrm{MHz}$ repetition rates and its pulse duration is approximately $0.5 \mathrm{~ns}$. Then it passes through the half-wave plate $\lambda / 2$ (Thorlabs AQWP05M-600) and is expanded through lenses f1-f2 to match the size of the aperture of the liquid crystal on silicon spatial light modulator SLM1 (Ha mamatsu X10468-02). $\lambda / 2$ rotates the depletion laser polarization so that it matches the orientation of the liquid crystal molecules of the SLM1. SLM1 is shaping the depletion beam into either 2D STED (Laguerre-Gaussian phase mask) or 3D STED (Top-Hat phase mask) beams. Pair of lenses $f 1^{\prime}-f 2^{\prime}$ is imaging the SLM1 plane onto the dichroic mirror DM2 plane. Glan laser polarizer (GLP) is blocking any polarizations not corresponding to the orientation of the liquid crystals in the SLM1. Depletion beam is reflected into the main beam-path through the dichroic mirror DM1 (Semrock FF720-SDi01) and at the DM2 (Chroma ZT647rdc-UF3) it is superimposed with the excitation beam. $\lambda / 2$ is used to rotate the polarization of the depletion beam. From the DM2 both depletion and excitation beams are co-propagating.

\section{Depletion beam path}

Excitation beam is generated by the $637 \mathrm{~nm}$ pulsed diode laser (PicoQuant LDH-P-C 640B). It operated at the same repetition rates that the depletion laser and has pulse width of 90ps. Excitation beam is coupled into the polarization maintaining single-mode fiber PMF using coupling lens FC. The output of the excitation beam has an elliptical shape and before coupling it has to be magnified over one axis using the anamorphic prism pair. This way the $75 \%$ of coupling efficiency can be obtained. $\lambda / 2$ rotates the polarization of the depletion beam, lens $f 3$ is used as collimator and such beam is then reflected from the SLM2 (Boulder Nonlinear Systems $512 \times 512$ ), which is used for correcting aberrations of the excitation beam. Lenses pair 
f4-f5 are imaging SLM2 plane onto the DM2 plane. DM2 is reflecting the excitation beam onto the main beam path and superimposes excitation beam with depletion beam.

\section{Main beam path}

Lenses $\mathrm{f} 6$ and $\mathrm{f} 7$ are imaging the $\mathrm{DM} 2$ plane onto the plane located between closely aligned pair of galvanometric mirrors GM (ScanLab Dynaxis XS) and lenses f8 and f9 image the GM plane onto the $16 \mathrm{kHz}$ resonant mirror RM (Electro-Optical Products Corp. SC-30) plane. Combination of GM and RM is used for scanning the sample - RM scans fast axis, GM scans slow axis. Lenses f10 and f11 are imaging RM plane onto the back focal plane of the microscope objective MO (Nikon CFI Plan Apo Lambda 100X Oil NA 1.45). MO is placed on the focusing piezo stage (Piezoconcept HS1.70) while sample is mounted on the motorized XY stage (ASI Imaging ASI S31121010FT).

\section{Imaging beam paths}

There are 3 imaging modes: brightfield transmission mode, quasi-widefield reflection mode and confocal fluorescent mode.

For imaging using a brightfield mode, sample is illuminated from top using white light diode. Transmitted light is directed onto the CCD camera using 92:8 pellicle beamsplitter BS1 and focused using tube lens $f 12$.

Reflected light, for imaging reflective objects such as gold beads, is de-scanned using RM and GM mirrors and then reflected using 92:8 pellicle beams splitter BS2. Then, lens f13 is focusing the reflected light through the widefield pinhole. Multi Pixel Photon Counter MPPC (Hamamatsu C13366).

Fluorescent light is de-scanned using the RM and GM mirrors, separated from the excitation beam by $B M 2$ and the separated from the depletion beam by $D M 1$. Lens $f 14$ is focusing the fluorescent signal into the multi-mode fiber MMF, which acts as a confocal pinhole. The size of 
the MMF is set to 0.8 Airy units. Bandpass emission filter EF (Semrock 676/37) is rejecting any remaining non-fluorescent signal.

\section{Pulse delay scheme}

The system uses pulsed diode lasers with the ability to trigger one another. In order to achieve the most efficient depletion, the depletion laser pulse needs to arrive right after the excitation laser pulse. We have experimentally estimated that the most efficient depletion was achieved when the depletion laser pulse arrived at the sample approx. 160ps after the excitation laser pulse. For easy pulse alignment we used an electronic picosecond pulse delayer (Picosecond Delayer, MPD, Bolzano, Italy) which allowed to precisely set the pulse delay with 10ps delay resolution.

\section{Image processing}

For image processing we used Matlab and the widely available scripts for Fourier Ring Correlation (code available in supplementary information of the Nieuwenhuizen et al. ${ }^{1}$ ) and BM3D (code available at the website of Department of Signal Processing of Tempere University of Technology uploaded by the authors of the original $B M 3 D^{2}$ ). For the estimation of the FRC resolution we need two identical images that only differ by the noise distribution. Since raw images collected by our STED microscope system already contain two mirrored images (due to the use of a resonant mirror for sample scanning), we only need to find the correct translation between them. For this, we use the Fourier cross correlation algorithm, ${ }^{3}$ which finds the translation between two images with subpixel resolution. This step is very important, as FRC highly depends on a lack of drift between images. ${ }^{4}$ After removing all drift, the FRC is calculated. BM3D denoising was performed on the raw images using the $\sigma$ as shown in main text and the normal profile setting. 


\section{References}

1. Nieuwenhuizen, R. P.; Lidke, K. A.; Bates, M.; Puig, D. L.; Grünwald, D.; Stallinga, S.; Rieger, B. Measuring Image Resolution in Optical Nanoscopy. Nature Methods 2013, 10, 557-562.

2. Dabov, K.; Foi, A.; Katkovnik, V. Image Denoising by Sparse 3D Transformation-Domain Collaborative Filtering. IEEE Transactions on Image Processing 2007, 16, 2080-2095.

3. Guizar-Sicairos, M.; Thurman, S. T.; Fienup, J. R. Efficient Subpixel Image Registration Algorithms. Optics letters 2008, 33, 156-158.

4. Tortarolo, G.; Castello, M.; Diaspro, A.; Koho, S.; Vicidomini, G. Evaluating Image Resolution in Stimulated Emission Depletion Microscopy. Optica 2018, 5, 32-35. 